\title{
The Association of Chemokine Gene Polymorphisms with VKH and Behcet's Disease in a Chinese Han Population
}

\author{
Yang Huang, ${ }^{1}$ Hongsong Yu, ${ }^{1}$ Qingfeng Cao, ${ }^{1}$ Jing Deng, ${ }^{1}$ Xinyue Huang, \\ Aize Kijlstra, ${ }^{2}$ and Peizeng Yang ${ }^{1}$ \\ ${ }^{1}$ The First Affiliated Hospital of Chongqing Medical University, Chongqing Key Laboratory of \\ Ophthalmology and Chongqing Eye Institute, Chongqing, China \\ ${ }^{2}$ University Eye Clinic Maastricht, Maastricht, Netherlands
}

Correspondence should be addressed to Peizeng Yang; peizengycmu@126.com

Received 20 January 2017; Revised 4 April 2017; Accepted 12 April 2017; Published 14 May 2017

Academic Editor: Paolo Spagnolo

Copyright (c) 2017 Yang Huang et al. This is an open access article distributed under the Creative Commons Attribution License, which permits unrestricted use, distribution, and reproduction in any medium, provided the original work is properly cited.

\begin{abstract}
To investigate the association of chemokine gene polymorphisms and Behcet's disease (BD) and Vogt Koyanagi Harada (VKH) disease in a Chinese Han population. A case-control study was performed. Three hundred and seventy-one BD patients, $371 \mathrm{VKH}$ disease patients, and 605 healthy controls were recruited to determine genetic variants of 26 SNPs in 12 chemokine genes with iPLEX Gold genotyping assay and Sequenom MassARRAY or TaqMan SNP assays. In this study, $P_{\text {uncorr }}$ values showed a weak association of five SNPs of five genes in BD and three SNPs of three genes in VKH disease. However, after Bonferroni correction, the 26 investigated SNPs showed no significant differences in genetic variants, including genotype and allele frequencies, between $\mathrm{BD}$ or VKH disease patients and healthy individuals. Haplotype analysis for the chemokine genes showed a significant association with the TC haplotype of CXCL12 in VKH. Stratified gender analysis and genotype-phenotype analysis were conducted to analyze the association of the 26 SNPs of 12 chemokine genes with BD and VKH disease. However, no significant association was observed after Bonferroni correction. This study showed no association of 26 SNPs in 12 chemokine genes with both BD and VKH disease in a Chinese Han population.
\end{abstract}

\section{Introduction}

Uveitis is an intraocular inflammatory disease causing severe visual impairment worldwide [1]. In China, Behcet's disease (BD) and Vogt-Koyanagi Harada (VKH) disease have the highest incidence in uveitis entities. BD is a chronic, relapsing, multisystemic inflammatory disorder, and its classical clinical characters include oral aphthae, genital ulcers, and recurrent iridocyclitis with hypopyon, which is probably due to an autoimmune response [2]. VKH disease is a multisystem autoimmune disease with a hallmark of diffuse granulomatous uveitis accompanied with poliosis, vitiligo, alopecia, and central nervous system abnormalities [3]. Various genes have been demonstrated to be relevant to different types of uveitis, comprising HLA-B27, HLA-A29, HLA-B51, HLA-DR4, IL-10, STAT4, STAT3, and UBAC2 [4-6] which suggested genetic factors are involved in the occurrence and development of uveitis.
Chemokines are a class of proinflammatory cytokines that are able to attract and activate the migration of circulating leukocytes under both physiological and pathological conditions [7]. According to the related structure and function, four subfamilies of human chemokines are classified: CC chemokines, CXC chemokines, CX3C family, and C family. Previous studies showed that chemokines are involved in various inflammatory and autoimmune diseases $[8,9]$. Chemokines also contribute to the pathogenesis of uveitis, and previous researches showed that a higher chemokine production might be responsible for the more severe clinical manifestations in Behcet's disease [10]. A comparison of Japanese VKH disease patients with controls indicated a dramatic decrease in the chemokine CSF-CCL2/MCP-1 [11].

Genetic variations of chemokine genes have been demonstrated responsible for the induction of chronic inflammation [7]. RANTES (CCL5) is associated with diabetes mellitus type 1 both genetically and functionally [12]. In the onset 
and development of childhood Idiopathic Thrombocytopenic Purpura, the polymorphism of SDF-1 (CXCL12) gene may be implicated [13]. Intron 1 of the CXCL9 gene (rs2276886) polymorphism may be closely related to pediatric Crohn's disease [14]. Among Chinese Han individuals, genetic variations of CXCL12-3 $3^{\prime}$ G801A are involved in the pathogenesis of systemic lupus erythematosus [15]. Only few studies have analyzed the association of uveitis with chemokine gene polymorphisms. In Caucasian patients with HLA-B27 associated acute anterior uveitis, the CCL2-2518G allele was found significantly increased [16] and IL-8 (CXCL8) gene polymorphisms may affect susceptibility to BD in Turkey [17]. However, the association between other chemokine gene polymorphisms with uveitis is largely unknown and has been addressed recently by our group. Earlier we reported that CCL2 polymorphisms were protective for BD [18]. In this study, we expanded the amount of chemokines SNPs and also included VKH disease patients. The results show that none of the other chemokine genes polymorphisms showed an association with $\mathrm{BD}$ or VKH disease in the Chinese Han population.

\section{Material and Methods}

2.1. Study Population. Our study recruited $371 \mathrm{BD}$ and 371 $\mathrm{VKH}$ disease patients and 605 healthy individuals which are all from Chinese Han population in the First Affiliated Hospital of Chongqing Medical University from January 2009 to April 2015 (Chongqing, China). According to race (Chinese Han) and geography, patients and the controls were matched. Diagnosis for BD and VKH disease followed the standard of the International Study Group for BD [19] and First International Workshop for VKH disease [20], respectively. The local research ethics committee approved the study and all the recruited individuals signed informed consent before donating blood samples. The Declaration of Helsinki adhered to the tenets.

2.2. Single Nucleotide Polymorphism (SNP) Selection. Screening of target chemokine gene SNPs was according to previously published studies which showed a positive association with other autoimmune and inflammatory diseases. Linkage disequilibrium (LD) data from the Han Chinese Hap Map database were taken into account. Twenty-seven SNPs of twelve genes with a minor allele frequency $>0.05$ in Han Chinese were selected. These 27 SNPs in 12 chemokine genes, included 4 SNPs (rs1024610, rs1024611, rs13900, and rs4586) of CCL2 [21, 22], 5 SNPs (rs4251719, rs2306630, rs2107538, rs9355610, and rs2280788) of CCL5 [12, 23, 24], 1 SNP (rs854680) of CCL16 [25], 2 SNPs (rs223828 and rs223895) of CCL17 [26-28], 3 SNPs (rs951005, rs2492358, and rs2812378) of CCL21 [29-31], 1 SNP (rs4359426) of CCL22 [32], 2 SNPs (rs2302004 and rs2302005) of CCL24 [33], 3 SNPs (rs2227306, rs2227543, and rs4694178) of CXCL8 [34], 2 SNPs (rs2276886 and rs2869460) of CXCL9 [14, 35], 1 SNP (rs2869462) of CXCL10 [35], 2 SNPs (rs1801157 and rs2839693) of CXCL12 [13, 15], and 1 SNP (rs2277680) of CXCL16 [36]. We excluded rs1024611 of CCL2, since a study concerning this gene had been reported previously by our group [18].

2.3. DNA Extraction and Genotyping. Peripheral blood of the three experimental groups including $\mathrm{BD}$ and $\mathrm{VKH}$ disease patients and the controls was subjected to genomic DNA extraction with the QIAmp DNA Blood Mini Kit (Qiagen Inc., Valencia, CA, USA) and the DNA was stored at $-80^{\circ} \mathrm{C}$. The Applied Biosystems 7500 Real-Time PCR system was utilized to genotype CCL17/rs223828 (TagMan assay ID: C_30530263_10) by the TaqMan SNP Genotyping Assay (Applied Biosystems, Foster City, CA, USA). Genotype identification of the other 25 SNPs was conducted with the iPLEX Gold genotyping assay and Sequenom MassARRAY (Sequenom, CA, USA). Sequenom SNP Assay Design software version 3.0 was used to design primers of iPLEX reactions. Primer sequences used were shown in Table 1. The protocol and experimental requirements were performed strictly based on the instructions.

2.4. Statistical Analysis. Hardy-Weinberg equilibrium (HWE) analysis was carried out by the Chi-square $\left(\chi^{2}\right)$ test in healthy samples while the genotype frequency was estimated by direct counting. No SNP significantly deviated from $\operatorname{HWE}(P>0.05)$. Fisher's exact test or $\chi^{2}$ test was applied to evaluate the differences in allele and genotype frequencies of all SNPs between patients and healthy controls using SPSS (version 17.0; SPSS Inc., Chicago, IL). The Bonferroni method was conducted to perform correction for multiple comparisons whereby the $P$ value was multiplied with the number of comparisons $(P$ corrected $(P c))$. It was considered to be significant when $P c<0.05$. In those genes having more than one SNP we also performed a haplotype analysis. Haplotypes with a frequency of 0.03 or larger were included in the analysis $[37,38] . P$ values for haplotypes were multiplied with the number of haplotypes in each gene. $P c<0.05$ was considered as significant. Gene-gene interaction analysis was performed using MDR software (MDR 3.0.2 obtained from https://sourceforge.net/projects/ $\mathrm{mdr} /)$.

\section{Results}

3.1. Clinical Features. The demographics and clinical symptoms of $\mathrm{BD}$ and $\mathrm{VKH}$ disease and demographics of controls are all showed in Table 2 . The healthy cohort is comprised of 321 men and 284 women, who were on average $38.6 \pm 11.1$ years old. The BD patients consisted of 371 subjects ( 326 men and 45 women), $33.2 \pm 8.4$ years old on average. The VKH disease group contained 371 subjects (204 men and 167 women), and the patients were on average $39.8 \pm 13.9$ years old.

3.2. Chemokine Genotyping Results. Twenty-six SNPs covering 12 chemokine genes (CCL2, CCL5, CCL16, CCL17, CCL21, CCL22, CCL24, CXCL8, CXCL9, CXCL10, CXCL12, and CXCL16) were genotyped successfully and all SNPs of controls met the Hardy-Weinberg equilibrium. There was no significant difference in allelic and genotypic frequencies for all the 26 SNPs in the patients of $\mathrm{BD}$ or $\mathrm{VKH}$ 


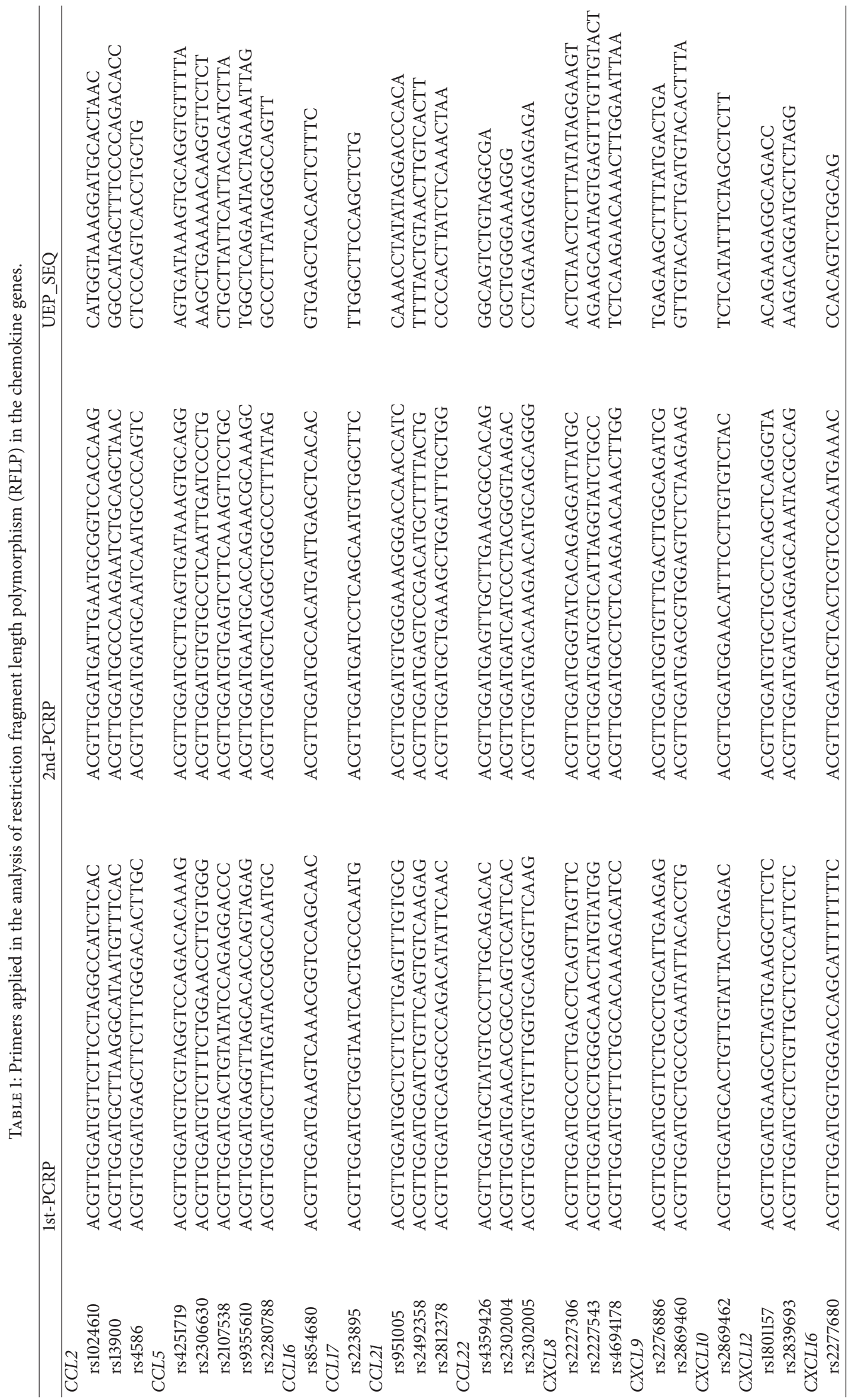


TABLE 2: Clinical features, age, and sex distribution of patients and controls.

\begin{tabular}{|c|c|c|}
\hline Clinical features & Total & $\%$ \\
\hline Patients with $B D$ & 371 & \\
\hline Mean age $\pm S D$ & $33.2 \pm 8.4$ & \\
\hline Male & 326 & 87.9 \\
\hline Female & 45 & 12.1 \\
\hline Uveitis & 358 & 96.5 \\
\hline Oral ulcer & 349 & 94 \\
\hline Genital ulcer & 208 & 56.1 \\
\hline Skin lesion & 272 & 73.3 \\
\hline Arthritis & 53 & 14.3 \\
\hline Pathergy reaction & 8 & 2.2 \\
\hline Patients with VKH disease & 371 & \\
\hline Mean age $\pm S D$ & $39.8 \pm 13.9$ & \\
\hline Male & 204 & 55 \\
\hline Female & 167 & 45 \\
\hline Sunset glow fundus & 182 & 49 \\
\hline Headache & 157 & 42.3 \\
\hline Tinnitus & 146 & 39.4 \\
\hline Vitiligo & 123 & 33.2 \\
\hline Alopecia & 136 & 36.7 \\
\hline Gray hair & 58 & 15.6 \\
\hline Controls & 605 & \\
\hline Mean age $\pm S D$ & $38.6 \pm 11.1$ & \\
\hline Male & 321 & 53.1 \\
\hline Female & 284 & 46.9 \\
\hline
\end{tabular}

$\mathrm{BD}=$ Behcet's disease, $\mathrm{SD}=$ standard deviation; $\mathrm{VKH}=$ Vogt-KoyanagiHarada.

disease compared with the controls (Supplemental Tables 1 and 2 in Supplementary Material available online at https://doi.org/10.1155/2017/1274960). However, $P_{\text {uncorr }}$ values showed a weak association of five SNPs of five genes in BD and three SNPs of three genes in VKH disease (Tables 3 and 4). Compared with $\mathrm{BD}$ patients, the frequency of the CCL5/rs2107538 CC genotype $(P=0.048$, OR $=1.309$, and $95 \% \mathrm{CI}=1.002-1.710)$ was decreased and the CCL5/rs2107538 CT genotype frequency $(P=0.042, \mathrm{OR}=0.76$, and $95 \%$ $\mathrm{CI}=0.583-0.991)$ was increased in the controls. A higher frequency of CCL17/rs223828 C allele $(P=0.028, \mathrm{OR}=$ 1.582 , and $95 \% \mathrm{CI}=1.048-2.389)$ and a lower TT genotype frequency $(P=0.020, \mathrm{OR}=0.795$, and $95 \% \mathrm{CI}=0.655-0.965)$ were found in the patients of $\mathrm{BD}$. In CCL22/rs4359426, the frequency of the AA genotype $(P=0.012$, OR $=3.071$, and $95 \% \mathrm{CI}=1.227-7.685)$ was increased in BD patients. The CXCL10/rs2869462 showed an increased frequency of the CC genotype and $\mathrm{C}$ allele $(P=0.034, \mathrm{OR}=1.347$, and $95 \% \mathrm{CI}=$ 1.023-1.774; $P=0.006, \mathrm{OR}=1.313$, and $95 \% \mathrm{CI}=1.081-1.595$, resp.), and CXCL12/rs1801157 showed a decreased frequency of the CT genotype $(P=0.047, \mathrm{OR}=0.761$, and $95 \% \mathrm{CI}=$ 0.581-0.996) in BD patients. In VKH disease patients, there was an increased frequency of the CCL5/rs9355610 A allele
$(P=0.029, \mathrm{OR}=0.805$, and $95 \% \mathrm{CI}=0.662-0.979)$ and CXCL8/rs2227543 CT genotype $(P=0.016, \mathrm{OR}=0.72$, and $95 \% \mathrm{CI}=0.552-0.940)$. In CXCL12/rs1801157, a weak association was detected in the $\mathrm{C}$ allele and CC and CT genotype in $\mathrm{VKH}$ disease $(P=0.01, \mathrm{OR}=1.327$, and $95 \%$ $\mathrm{CI}=1.069-1.647 ; P=0.00118, \mathrm{OR}=1.556$, and $95 \% \mathrm{CI}=$ $1.190-2.033 ; P=8.463 \times 10^{-4}, \mathrm{OR}=0.627$, and $95 \% \mathrm{CI}=$ $0.476-0.826$ ). However, after correction for multiple comparisons, all associations described above lost statistical significance.

3.3. Haplotype Analysis. The haplotypes of chemokine genes (CCL2, CCL5, CCL17, CCL21, CCL24, CXCL8, CXCL9, and CXCL12) having more than one SNP were analyzed using the website http://analysis.bio-x.cn/myAnalysis.php. The haplotype TC of the CXCL12 gene including two SNPs (rs1801157 and rs2839693) showed a significant association with $\mathrm{VKH}$ $(P=0.008, P c=0.032, \mathrm{OR}=0.745$, and $95 \% \mathrm{CI}=0.599$ 0.927) (Table 5) compared with healthy controls. The other tested haplotypes failed to show an association with either BD or VKH.

3.4. Stratified Analysis according to Gender and Main Clinical Manifestations of $B D$ and VKH Disease. Stratified analyses were conducted to investigate whether the 26 SNPs have an association with gender and the primary clinical features in $\mathrm{BD}$ and $\mathrm{VKH}$ disease. BD in our population is more often seen in males and we therefore believe that a gender analysis might also be involved in the genetic predisposition to this disease and a previous study showed that chemokine gene SNPs of both CCL2 gene and CCL5 were more prevalent in males than females with BD [39]. To further confirm whether gender could influence genotype and allele frequencies in both diseases we performed the gender stratified study in these two diseases. We chose clinical manifestations with the frequency of approximately $50 \%$. These included the presence of genital ulcers in BD and sunset glow fundus in VKH disease, respectively. Following Bonferroni correction, no association was observed after stratification by gender (Supplemental Tables 3 and 4). Also no significant differences were detected in these SNPs after stratifying VKH with sunset glow fundus or not. Additionally, no significant association was observed when BD was stratified by genital ulcer. MDR analysis was performed to test the gene-gene (epistatic effect) analysis interaction among 26 SNPs of 12 chemokine genes and this analysis showed that no gene-gene interaction existed in these two diseases. (Supplemental Tables 5 and 6).

\section{Discussion}

In the present study, we described the genotyping of $371 \mathrm{BD}$ and $371 \mathrm{VKH}$ patients and 605 controls from Chinese Han population for twenty-six SNPs in twelve chemokine genes. The AA genotype of MCP-1-2518 (rs1024611/CCL2) has been shown by our previous research to have a protective association with BD in the Chinese Han population [18]. Extension of this latter study with other chemokine polymorphisms and including another uveitis entity (VKH) did not reveal any new associations, although haplotype analysis did show that 
TABLE 3: Genotype and allele frequencies of five chemokine genes' polymorphism in BD and healthy controls.

\begin{tabular}{|c|c|c|c|c|c|c|c|c|}
\hline Gene & SNP & & $\mathrm{BD} n(\%)$ & Controls $n(\%)$ & $P$ value & $P c$ value & OR & $95 \%$ CI \\
\hline \multirow{6}{*}{ CCL5 } & \multirow{6}{*}{ rs 2107538} & Total sample & 368 & 556 & & & & \\
\hline & & $\mathrm{CC}$ & $165(0.448)$ & $213(0.383)$ & 0.048 & NS & 1.309 & $1.002-1.710$ \\
\hline & & CT & $155(0.421)$ & $272(0.489)$ & 0.042 & NS & 0.760 & $0.583-0.991$ \\
\hline & & $\mathrm{TT}$ & $48(0.130)$ & $71(0.128)$ & 0.903 & NS & 1.025 & $0.692-1.517$ \\
\hline & & $\mathrm{C}$ & $485(0.659)$ & $698(0.628)$ & 0.170 & NS & 1.146 & $0.943-1.393$ \\
\hline & & $\mathrm{T}$ & $251(0.341)$ & $414(0.372)$ & & & 0.873 & $0.718-1.060$ \\
\hline \multirow{6}{*}{ CCL17 } & \multirow{6}{*}{$r s 223828$} & Total sample & 371 & 604 & & & & \\
\hline & & $\mathrm{CC}$ & $155(0.418)$ & $287(0.575)$ & 0.081 & NS & 0.793 & $0.611-1.029$ \\
\hline & & $\mathrm{CT}$ & $167(0.450)$ & $264(0.437)$ & 0.690 & NS & 1.054 & $0.813-1.368$ \\
\hline & & $\mathrm{TT}$ & $49(0.132)$ & $53(0.088)$ & 0.028 & NS & 1.582 & $1.048-2.389$ \\
\hline & & $\mathrm{C}$ & $477(0.643)$ & $838(0.694)$ & 0.020 & NS & 0.795 & $0.655-0.965$ \\
\hline & & $\mathrm{T}$ & $265(0.357)$ & $370(0.306)$ & & & & \\
\hline \multirow{6}{*}{ CCL22 } & \multirow{6}{*}{$r s 4359426$} & Total sample & 365 & 546 & & & & \\
\hline & & AA & $14(0.038)$ & $7(0.013)$ & 0.012 & NS & 3.071 & $1.227-7.685$ \\
\hline & & $\mathrm{AC}$ & $93(0.255)$ & $134(0.245)$ & 0.749 & NS & 1.051 & $0.774-1.427$ \\
\hline & & $\mathrm{CC}$ & $258(0.707)$ & $405(0.742)$ & 0.246 & NS & 0.839 & $0.624-1.128$ \\
\hline & & A & $121(0.166)$ & $148(0.136)$ & 0.075 & NS & 1.267 & $0.976-1.645$ \\
\hline & & $\mathrm{C}$ & $609(0.834)$ & $944(0.864)$ & & & 0.789 & $0.608-1.024$ \\
\hline \multirow{6}{*}{ CXCL10 } & \multirow{6}{*}{$r s 2869462$} & Total sample & 360 & 535 & & & & \\
\hline & & $\mathrm{CC}$ & $149(0.414)$ & $184(0.344)$ & 0.034 & NS & 1.347 & $1.023-1.774$ \\
\hline & & CG & $160(0.444)$ & $243(0.454)$ & 0.773 & NS & 0.961 & $0.735-1.258$ \\
\hline & & GG & $51(0.142)$ & $108(0.202)$ & 0.210 & NS & 0.653 & $0.454-0.939$ \\
\hline & & $\mathrm{C}$ & $458(0.636)$ & $611(0.571)$ & 0.006 & NS & 1.313 & $1.081-1.595$ \\
\hline & & G & $262(0.364)$ & $459(0.429)$ & & & 0.761 & $0.627-0.925$ \\
\hline \multirow{6}{*}{ CXCL12 } & \multirow{6}{*}{ rs1801157 } & Total sample & 371 & 547 & & & & \\
\hline & & $\mathrm{CC}$ & $207(0.558)$ & $271(0.495)$ & 0.063 & NS & 1.285 & $0.987-1.675$ \\
\hline & & $\mathrm{CT}$ & $139(0.375)$ & $241(0.441)$ & 0.047 & NS & 0.761 & $0.581-0.996$ \\
\hline & & $\mathrm{TT}$ & $25(0.067)$ & $35(0.064)$ & 0.838 & NS & 1.057 & $0.621-1.798$ \\
\hline & & $\mathrm{C}$ & $553(0.745)$ & $783(0.716)$ & 0.163 & NS & 1.162 & $0.941-1.435$ \\
\hline & & $\mathrm{T}$ & $189(0.255)$ & $311(0.284)$ & & & 0.860 & $0.697-1.063$ \\
\hline
\end{tabular}

TABLE 4: Genotype and allele frequencies of three chemokine genes' polymorphism in VKH and healthy controls.

\begin{tabular}{|c|c|c|c|c|c|c|c|c|}
\hline Gene & SNP & & $\mathrm{VKH} n(\%)$ & Controls $n(\%)$ & $P$ value & $P c$ value & OR & $95 \% \mathrm{CI}$ \\
\hline \multirow{6}{*}{ CCL5 } & \multirow{6}{*}{ rs9355610 } & Total sample & 370 & 555 & & & & \\
\hline & & $\mathrm{AA}$ & $97(0.262)$ & $138(0.249)$ & 0.644 & NS & 1.074 & $0.794-1.451$ \\
\hline & & AG & $188(0.508)$ & $273(0.492)$ & 0.629 & NS & 1.067 & $0.820-1.388$ \\
\hline & & GG & $85(0.230)$ & $144(0.259)$ & 0.305 & NS & 0.851 & $0.626-1.158$ \\
\hline & & $\mathrm{A}$ & $282(0.516)$ & $549(0.495)$ & 0.029 & NS & 0.805 & $0.662-0.979$ \\
\hline & & G & $358(0.484)$ & $561(0.505)$ & & & 1.242 & $1.022-1.511$ \\
\hline \multirow{6}{*}{ CXCL8 } & \multirow{6}{*}{ rs 2227543} & Total sample & 364 & 552 & & & & \\
\hline & & CC & $146(0.401)$ & $187(0.339)$ & 0.055 & NS & 1.307 & 0.994-1.719 \\
\hline & & CT & $155(0.426)$ & $280(0.507)$ & 0.016 & NS & 0.720 & $0.552-0.940$ \\
\hline & & TT & $63(0.173)$ & $85(0.154)$ & 0.442 & NS & 1.15 & $0.805-1.643$ \\
\hline & & $\mathrm{C}$ & $447(0.614)$ & $654(0.592)$ & 0.355 & NS & 1.095 & $0.904-1.326$ \\
\hline & & $\mathrm{T}$ & $281(0.386)$ & $450(0.408)$ & & & 0.914 & $0.754-1.106$ \\
\hline \multirow{6}{*}{ CXCL12 } & \multirow{6}{*}{ rs1801157 } & Total sample & 368 & 547 & & & & \\
\hline & & $\mathrm{CC}$ & $223(0.605)$ & $271(0.495)$ & 0.001 & NS & 1.566 & $1.198-2.048$ \\
\hline & & $\mathrm{CT}$ & $122(0.331)$ & $241(0.441)$ & $9.443 * 10^{-4}$ & NS & 0.637 & $0.478-0.829$ \\
\hline & & TT & $23(0.062)$ & $35(0.064)$ & 0.928 & NS & 0.975 & $0.566-1.679$ \\
\hline & & $\mathrm{C}$ & $568(0.771)$ & $783(0.716)$ & 0.008 & NS & 1.343 & $1.081-1.668$ \\
\hline & & $\mathrm{T}$ & $168(0.228)$ & $311(0.284)$ & & & 0.745 & $0.600-0.925$ \\
\hline
\end{tabular}


TABLE 5: Haplotype analysis of CXCL12 gene polymorphisms in VKH.

\begin{tabular}{|c|c|c|c|c|c|c|c|}
\hline Haplotype & VKH (\%) & Control (\%) & $\chi^{2}$ & $P$ value & $P c$ value & OR & $95 \%$ CI \\
\hline CC & $466.03(0.633)$ & $630.97(0.590)$ & 2.875 & 0.089 & NS & 1.182 & $0.974-1.435$ \\
\hline $\mathrm{CT}$ & $104.97(0.143)$ & $135.03(0.126)$ & 0.916 & 0.338 & NS & 1.143 & $0.869-1.504$ \\
\hline TC & $164.97(0.224)$ & $297.03(0.278)$ & 6.959 & 0.008 & 0.032 & 0.745 & $0.599-0.927$ \\
\hline TT & $0.03(0.000)$ & $6.97(0.007)$ & & & & & \\
\hline
\end{tabular}

the haplotype TC of the CXCL12 gene including rs1801157 and rs2839693 shows a significant association with VKH.

Behcet's disease, which is considered an autoinflammatory disorder, is characterized by posterior or generalized uveitis with a chronic nature and with recurrent episodes [2]. VKH disease is considered as a multisystem disorder caused by an autoimmune response against melanocyte associated antigens [3]. The attraction of leukocytes to tissues is an important feature of inflammation and is mediated by the local release of chemokines [40]. Genetic variation in the genes encoding these chemokines may affect their function and may be associated with disease predisposition. Several studies have reported investigations concerning the association of a limited number of chemokine genetic variations in patients with different uveitis entities [21,39,41], but a large scale analysis on chemokine gene associations with $\mathrm{BD}$ or VKH disease has not been reported.

Despite the fact that the 26 SNPs chosen for our study have been proved to be associated with several other immune-mediated diseases, we did not detect any significant association between these SNPs and the two uveitis entities, $\mathrm{BD}$ or $\mathrm{VKH}$ disease. An exception is the association of the haplotype TC of the CXCL12 gene including rs1801157 and rs2839693 with VKH, which suggests that CXCL12 polymorphisms might be a risk factor contributing to VKH disease in the Chinese population. Our study confirms earlier data presenting the absence of an association between the chemokine genes rs1024610/CCL2 and rs2280788/rs2107538/CCL5 with Behcet's disease or retinal vasculitis in patients from UK [39]. Others showed that the frequency of the T allele of MCP163555 (rs1024610/CCL2) was significantly associated with idiopathic anterior uveitis in Caucasian patients [21], which could not be shown in the uveitis entities we studied. This discrepancy may be due to differences in the uveitis entity studied or due to ethnic effects.

Selection of candidate SNPs is a crucial step for a gene variation study. In our study, 26 SNPs covering 12 chemokine genes (CCL2, CCL5, CCL16, CCL17, CCL21, CCL22, CCL24, CXCL8, CXCL9, CXCL10, CXCL12, and CXCL16) were selected on the basis of earlier association studies in autoimmune diseases, including type 1 diabetes [12], pediatric Crohn's disease [14], and systemic lupus erythematosus [15]. It should be noted that composition and stratification of recruiting population may conclude to different results of an association study. To make sure that our data and results were valid, a series of efforts were made. First of all, the BD patients were diagnosed in strict accordance with the criteria of the International Study Group for BD while the VKH patients were diagnosed in strict accordance with the First International Workshop criteria of VKH disease. Any doubt or uncertainty in patient diagnosis is not allowed. Beyond that, to avoid ethnic bias, $\mathrm{BD}$ and $\mathrm{VKH}$ disease patients from other ethnic populations other than Chinese Han population were excluded.

Our study has several limitations. We only chose SNPs that have been previously reported to be related to autoimmune and inflammatory diseases, thus other unknown SNPs of chemokine genes with potential association with $\mathrm{BD}$ and VKH disease might be excluded. Furthermore, we studied only two common types of uveitis, with all the participants from Chinese Han population. Association of chemokine genes with other types of uveitis or different ethnic populations might also exist and awaits further investigation.

\section{Conclusions}

A large scale analysis of the role of chemokine genes only shows an association of CCL2 with BD but no effect on predisposition to VKH in Chinese Han population. The haplotype TC of the CXCL12 gene however did show a significant association with VKH compared with healthy controls.

\section{Conflicts of Interest}

The authors declare no conflicts of interest.

\section{Acknowledgments}

The authors would like to thank all donors enrolled in the present study. This work was supported by Natural Science Foundation Major International (Regional) Joint Research Project (81320108009), National Natural Science Foundation Project (31370893), Chongqing Key Laboratory of Ophthalmology (CSTC, 2008CA5003), National Key Clinical Specialties Construction Program of China, Key Project of Health Bureau of Chongqing (2012-1-003), Chongqing Science \& Technology Platform and Base Construction Program (cstc2014pt-sy10002), and the Major Research Development Program of China (2016YFC0904000).

\section{References}

[1] A. Rothova, M. S. A. Suttorp-van Schulten, W. Frits Treffers, and A. Kijlstra, "Causes and frequency of blindness in patients with intraocular inflammatory disease," The British Journal of Ophthalmology, vol. 80, no. 4, pp. 332-336, 1996.

[2] P. Yang, W. Fang, Q. Meng, Y. Ren, L. Xing, and A. Kijlstra, "Clinical features of Chinese patients with Behçet's disease," Ophthalmology, vol. 115, no. 2, pp. 312-318.e4, 2008. 
[3] P. Yang, Y. Ren, W. Fang, B. Li, A. Kijlstra, and Q. Meng, "Clinical characteristics of Vogt-Koyanagi-Harada syndrome in Chinese patients," Ophthalmology, vol. 114, no. 3, pp. 606-614, 2007.

[4] S. Hou, A. Kijlstra, and P. Yang, "Molecular genetic advances in uveitis," Progress in Molecular Biology and Translational Science, vol. 134, pp. 283-298, 2015.

[5] L. Du, A. Kijlstra, and P. Yang, "Immune response genes in uveitis," Ocular Immunology and Inflammation, vol. 17, no. 4, pp. 249-256, 2009.

[6] H. Yu, M. Zheng, L. Zhang et al., "Identification of susceptibility SNPs in IL10 and IL23R-IL12RB2 for Behçet's disease in Han Chinese," Journal of Allergy and Clinical Immunology, 2016.

[7] J. Guergnon and C. Combadière, "Role of chemokines polymorphisms in diseases," Immunology Letters, vol. 145, no. 1-2, pp. 15$22,2012$.

[8] I. F. Charo and R. M. Ransohoff, "The many roles of chemokines and chemokine receptors in inflammation," The New England Journal of Medicine, vol. 354, no. 6, pp. 610-621, 2006.

[9] W. Cheng and G. Chen, "Chemokines and chemokine receptors in multiple sclerosis," Mediators of Inflammation, vol. 2014, Article ID 659206, 8 pages, 2014.

[10] A. M. Abu El-Asrar, S. S. Al-Obeidan, D. Kangave et al., "CXC chemokine expression profiles in aqueous humor of patients with different clinical entities of endogenous uveitis," Immunobiology, vol. 216, no. 9, pp. 1004-1009, 2011.

[11] I. Miyazawa, T. Abe, K. Narikawa et al., "Chemokine profile in the cerebrospinal fluid and serum of Vogt-Koyanagi-Harada disease," Journal of Neuroimmunology, vol. 158, no. 1-2, pp. $240-$ 244, 2005.

[12] A. Zhernakova, B. Z. Alizadeh, P. Eerligh et al., "Genetic variants of RANTES are associated with serum RANTES level and protection for type 1 diabetes," Genes and Immunity, vol. 7, no. 7, pp. 544-549, 2006.

[13] F.-C. Ku, C.-R. Tsai, J.-D. Wang, C. H. Wang, T.-K. Chang, and W.-L. Hwang, "Stromal-derived factor-1 gene variations in pediatric patients with primary immune thrombocytopenia," European Journal of Haematology, vol. 90, no. 1, pp. 25-30, 2013.

[14] M. Lacher, R. Kappler, S. Berkholz, H. Baurecht, D. von Schweinitz, and S. Koletzko, "Association of a CXCL9 polymorphism with pediatric Crohn's disease," Biochemical and Biophysical Research Communications, vol. 363, no. 3, pp. 701-707, 2007.

[15] F.-X. Wu, X.-Y. Luo, L.-J. Wu et al., "Association of chemokine CXCL12-3/G801A polymorphism with systemic lupus erythematosus in a Han Chinese population," Lupus, vol. 21, no. 6, pp. 604-610, 2012.

[16] B. J. Wegscheider, M. Weger, W. Renner et al., "Role of the CCL2/MCP-1 -2518A > G gene polymorphism in HLA-B27 associated uveitis," Molecular Vision, vol. 11, pp. 896-900, 2005.

[17] A. Atalay, S. Arikan, O. Ozturk et al., "The IL-8 gene polymorphisms in behçet's disease observed in denizli province of turkey," Immunological Investigations, vol. 45, no. 4, pp. 298-311, 2016.

[18] S. Hou, P. Yang, L. Du et al., "Monocyte chemoattractant protein-1-2518 A/G single nucleotide polymorphism in Chinese Han patients with ocular Behçet's disease," Human Immunology, vol. 71, no. 1, pp. 79-82, 2010.

[19] International Study Group for Behçet's Disease, "Criteria for diagnosis of Behçet's disease," The Lancet, vol. 335, no. 8697, pp. 1078-1080, 1990.
[20] R. W. Read, G. N. Holland, N. A. Rao et al., "Revised diagnostic criteria for Vogt-Koyanagi-Harada disease: report of an international committee on nomenclature," American Journal of Ophthalmology, vol. 131, no. 5, pp. 647-652, 2001.

[21] T. K. Yeo, M. A. Ahad, N.-W. Kuo et al., "Chemokine gene polymorphisms in idiopathic anterior uveitis," Cytokine, vol. 35, no. 1-2, pp. 29-35, 2006.

[22] R. Cáliz, L. M. Canet, C. B. Lupiañez et al., "Gender-specific effects of genetic variants within Th1 and Th17 cell-mediated immune response genes on the risk of developing rheumatoid arthritis," PLoS ONE, vol. 8, no. 8, Article ID e72732, 2013.

[23] X.-J. Chen, X.-H. Gong, N. Yan et al., "RNASET2 tag SNP but not CCR6 polymorphisms is associated with autoimmune thyroid diseases in the Chinese Han population," BMC Medical Genetics, vol. 16, no. 1, article 11, 2015.

[24] Z.-K. Xie, H. Zhao, J. Huang, and Z.-F. Xie, "The regulated upon activation normal T-cell expressed and secreted (RANTES) $-28 \mathrm{C} / \mathrm{G}$ and $-403 \mathrm{G} / \mathrm{A}$ polymorphisms and asthma risk: a metaanalysis," Molecular Diagnosis and Therapy, vol. 18, no. 5, pp. 523-531, 2014.

[25] T. Vyshkina, A. Sylvester, S. Sadiq, E. Bonilla, A. Perl, and B. Kalman, "CCL genes in multiple sclerosis and systemic lupus erythematosus," Journal of Neuroimmunology, vol. 200, no. 1-2, pp. 145-152, 2008.

[26] S. A. Fisher, A. Moody, M. M. Mirza et al., "Genetic variation at the chromosome 16 chemokine gene cluster: development of a strategy for association studies in complex disease," Annals of Human Genetics, vol. 67, no. 5, pp. 377-390, 2003.

[27] D. Galimberti, D. Scalabrini, C. Fenoglio et al., "Gender-specific influence of the chromosome 16 chemokine gene cluster on the susceptibility to Multiple Sclerosis," Journal of the Neurological Sciences, vol. 267, no. 1-2, pp. 86-90, 2008.

[28] F. Liu, Y. Ding, and W. Yin, "Association of single nucleotide polymorphisms in TARC/CCL17 gene with Kawasaki disease and its clinical characteristics," Zhongguo Dang Dai Er Ke Za Zhi, vol. 17, no. 7, pp. 668-671, 2015.

[29] S. Raychaudhuri, E. F. Remmers, A. T. Lee et al., "Common variants at CD40 and other loci confer risk of rheumatoid arthritis," Nature Genetics, vol. 40, no. 10, pp. 1216-1223, 2008.

[30] E. A. Stahl, S. Raychaudhuri, E. F. Remmers et al., "Genomewide association study meta-analysis identifies seven new rheumatoid arthritis risk loci," Nature Genetics, vol. 42, no. 6, pp. 508-514, 2010.

[31] S. Chen, Q. Wang, C. Y. Wu et al., "A single-nucleotide polymorphism of CCL21 rs951005 T>C is associated with susceptibility of polymyositis and such patients with interstitial lung disease in a Chinese Han population," Clinical and Experimental Rheumatology, vol. 33, no. 5, pp. 639-646, 2015.

[32] T. Hirota, H. Saeki, K. Tomita et al., "Variants of C-C motif chemokine 22 (CCL22) are associated with susceptibility to atopic dermatitis: case-control studies," PLOS ONE, vol. 6, no. 11, Article ID e26987, 2011.

[33] Y. R. Park, S. C. Choi, S. T. Lee, K. Kim, S. Chae, and H. Chung, "The association of eotaxin-2 and eotaxin-3 gene polymorphisms in a Korean population with ulcerative colitis," Experimental \& Molecular Medicine, vol. 37, no. 6, pp. 553-558, 2005.

[34] J.-S. Suh, W.-H. Hahn, and B.-S. Cho, "Polymorphisms of CXCL8 and its receptor CXCR2 contribute to the development and progression of childhood IgA nephropathy," Journal of Interferon and Cytokine Research, vol. 31, no. 3, pp. 309-315, 2011. 
[35] J. Zhang, E. Noguchi, O. Migita et al., "Association of a haplotype block spanning SDAD1 gene and CXC chemokine genes with allergic rhinitis," Journal of Allergy and Clinical Immunology, vol. 115, no. 3, pp. 548-554, 2005.

[36] J. Seiderer, J. Dambacher, D. Leistner et al., "Genotypephenotype analysis of the CXCL16 p.Ala181Val polymorphism in inflammatory bowel disease," Clinical Immunology, vol. 127, no. 1, pp. 49-55, 2008.

[37] Y. Y. Shi and L. He, "SHEsis, a powerful software platform for analyses of linkage disequilibrium, haplotype construction, and genetic association at polymorphism loci," Cell Research, vol. 15, no. 2, pp. 97-98, 2005.

[38] Z. Li, Z. Zhang, Z. He et al., "A partition-ligation-combinationsubdivision em algorithm for haplotype inference with multiallelic markers: update of the SHEsis (http://analysis.bio-x.cn)," Cell Research, vol. 19, no. 4, pp. 519-523, 2009.

[39] Y. Chen, R. W. Vaughan, E. Kondeatis et al., "Chemokine gene polymorphisms associate with gender in patients with uveitis," Tissue Antigens, vol. 63, no. 1, pp. 41-45, 2004.

[40] A. D. Luster, "Mechanisms of disease: chemokines chemotactic cytokines that mediate inflammation," The New England Journal of Medicine, vol. 338, no. 7, pp. 436-445, 1998.

[41] S.-K. Kim, W.-C. Jang, Y.-C. Ahn, S.-H. Lee, S.-S. Lee, and J.W. Hur, "Promoter -2518 single nucleotide polymorphism of monocyte chemoattractant protein-1 is associated with clinical severity in Behçet's disease," Inflammation Research, vol. 61, no. 6, pp. 541-545, 2012. 


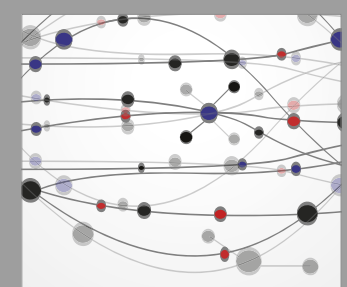

The Scientific World Journal
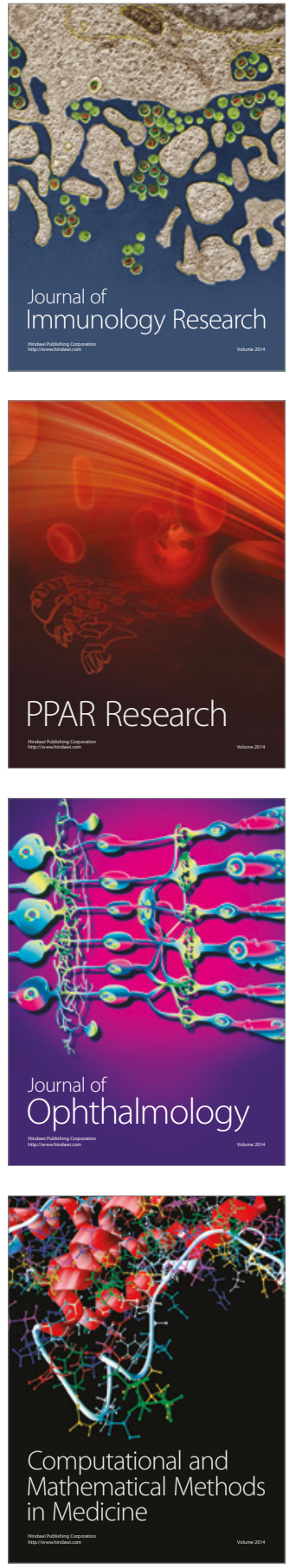

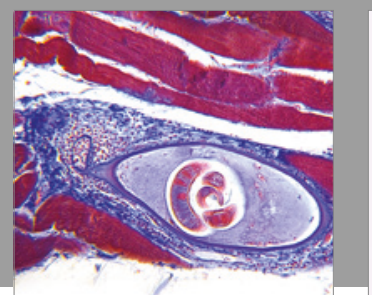

Gastroenterology Research and Practice
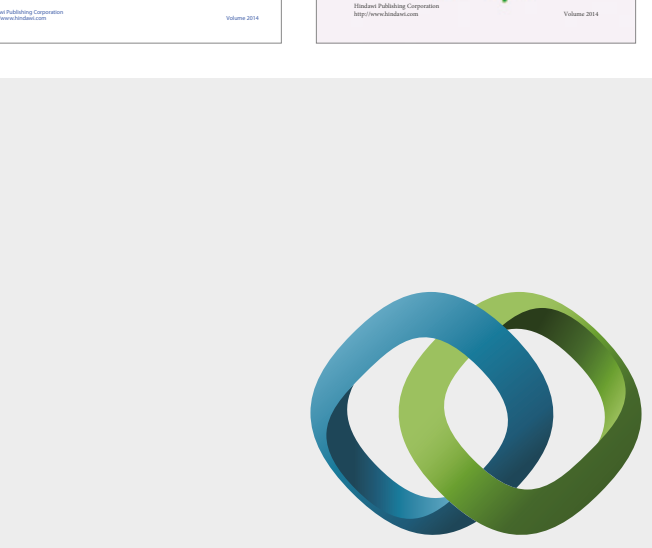

\section{Hindawi}

Submit your manuscripts at

https://www.hindawi.com
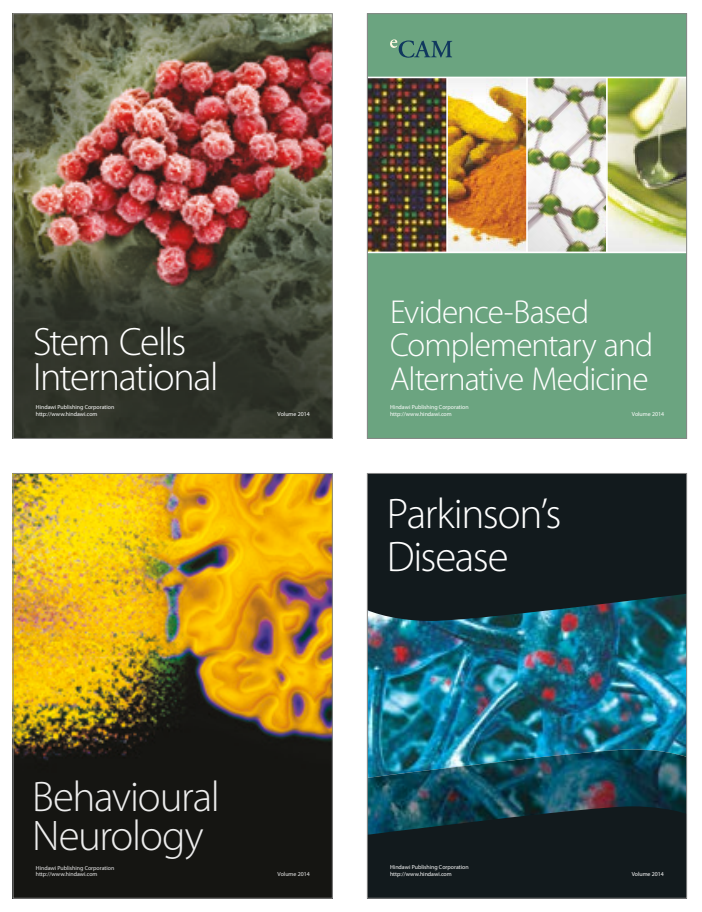
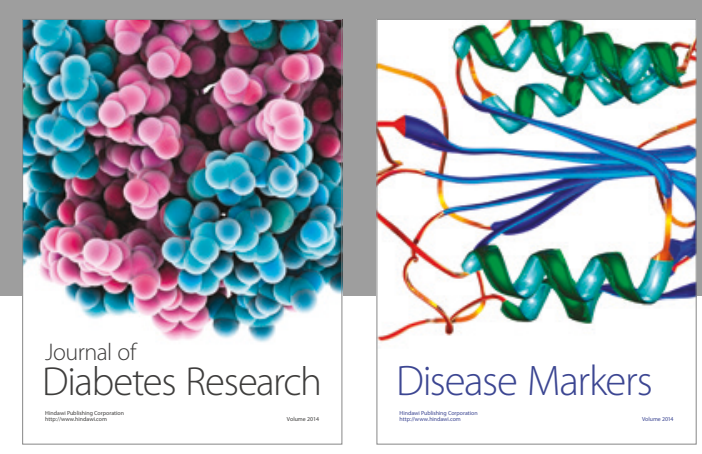

Disease Markers
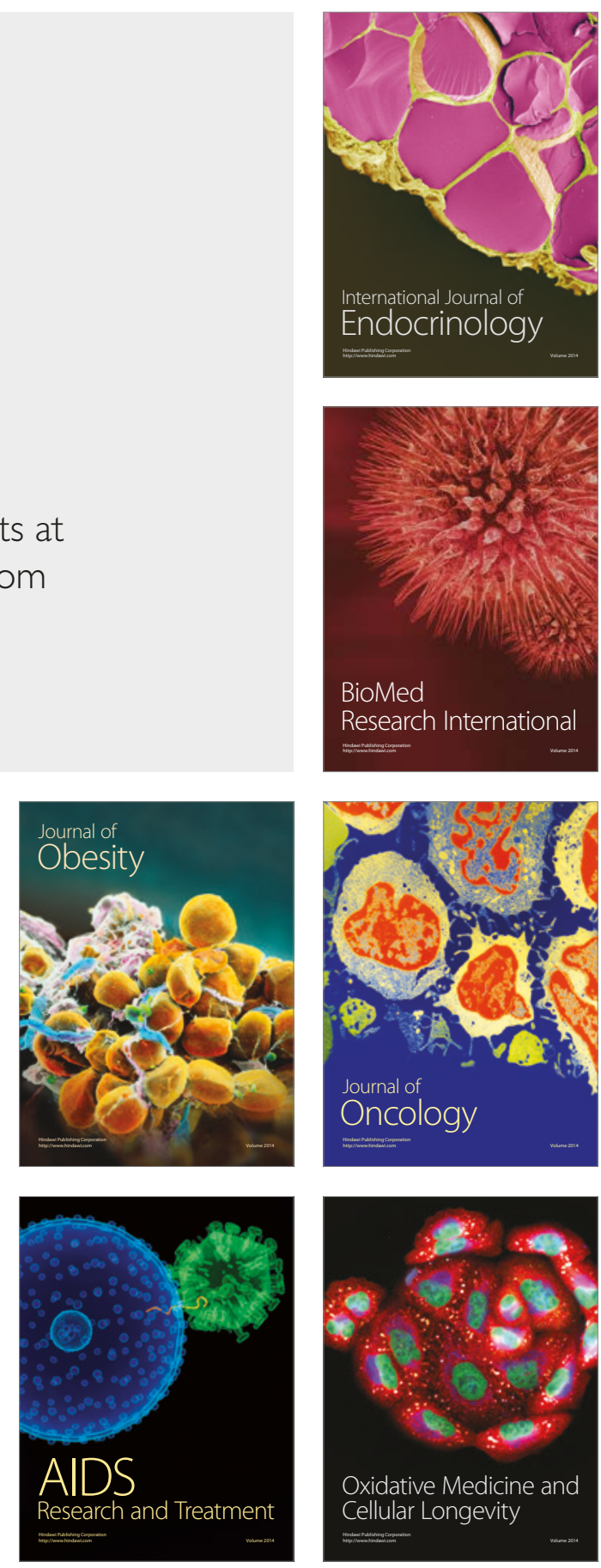